\title{
Clinical Predictors of Severe Cetuximab-Induced Rash: Observations from 933 Patients Enrolled in North Central Cancer Treatment Group Study N0147
}

\author{
Aminah Jatoi $^{\mathrm{a}}$ Erin M. Green ${ }^{\mathrm{a}}$ Kendrith M. Rowland, Jr. ${ }^{\mathrm{b}}$ Daniel J. Sargent ${ }^{\mathrm{a}}$ \\ Steven R. Alberts ${ }^{a}$ \\ a Mayo Clinic, Rochester, Minn., and ${ }^{b}$ Carle Cancer Center CCOP, Urbana, III., USA
}

\section{Key Words}

Cetuximab · Rash · Epidermal growth factor receptor

inhibitors $\cdot$ Colon cancer

\begin{abstract}
Objective: Epidermal growth factor receptor inhibitors can result in a severe rash in $5-10 \%$ of patients and can detract from quality of life. The objective of this study was to identify clinical predictors of severe rash in the hope of utilizing such factors in the design of future rash palliative and prevention trials. Methods: 933 cetuximab-treated patients enrolled on N0147, an adjuvant chemotherapy trial for colon cancer, were evaluated for clinical risk factors of severe rash. Results: Within this cohort, 50 patients (5\%) developed a severe rash (grade 3 ). More men compared to women developed such a rash: 34 (7\%) versus 16 (3\%) (multivariate odds ratio $=2.12 ; 95 \%$ confidence interval: $1.14-3.88 ; p=0.017)$. A greater number of younger patients ( $<70$ years of age) also developed a rash: $48(6 \%)$ versus $2(1 \%)$ (multivariate odds ratio $=0.21 ; 95 \%$ confidence interval: $0.05-0.88 ; p=0.032$ ). Race and performance score were not predictive. Conclusion: Men and younger patients are at greater risk for a severe cetuximab-induced rash although overall the risk is low. These observations are particularly important in designing future rash prevention and palliation trials.
\end{abstract}

Copyright $\odot 2009$ S. Karger AG, Basel
() 2009 S. Karger AG, Basel

Fax +41613061234 E-Mail karger@karger.ch www.karger.com www.karger.com/ocl
Epidermal growth factor receptor inhibitors are being used more often in cancer therapy; rash is their most common side effect [1]. This rash typically occurs on the face, trunk, and upper extremities within 1 week of starting cancer therapy; it can be severe in $5-10 \%$ of patients, reaching rates as high as $12 \%$ in patients receiving cetuximab and other chemotherapy $[1,2]$. Almost never fatal, this skin toxicity does, nonetheless, spawn considerable morbidity [3, 4]. Cutaneous pain and hypersensitivity, worries about appearance, and frustration from having to contend with this side effect - all detract from cancer patients' quality of life and translate into substantial morbidity [4]. This morbidity is all the more concerning because of limited preventive and palliative options [1, 5-7].

In view of this negative impact, the identification of clinical factors capable of predicting the development of a severe rash would be of value. Such predictors would enable healthcare providers to counsel patients about the prospect of a severe rash, thereby allowing patients to prepare themselves emotionally for this disfiguring, uncomfortable side effect. Perhaps of greater practical value, such predictors would also be important in designing clinical trials for rash prevention and palliation. To date, few studies have focused on factors associated with rash development although preliminary pharmacokinetic and pharmacogenomic models are starting to be explored [8].

Aminah Jatoi, MD

Mayo Clinic

200 First Street SW

Rochester, MN 55905 (USA)

Tel. +1 507284 3902, Fax +1 507284 1803, E-Mail jatoi.aminah@mayo.edu 
There remains a need for a clinically based approach to enable healthcare providers to identify patients at risk for severe rash development.

We therefore undertook the present study to identify such risk factors. Utilizing data from a large, multi-institutional ongoing adjuvant colon cancer clinical trial that tests cetuximab, we report here on the percentage of patients who developed a severe rash from an epidermal growth factor receptor inhibitor as well as on the clinical factors predictive of severe rash development.

\section{Methods}

\section{Overview}

This study represents a preliminary, secondary analysis of data derived from N0147, a North Central Cancer Treatment Group phase III study that is ongoing at the time of this report. This study tests adjuvant chemotherapy in patients with surgically resected stage III colon cancer [9]. All institutions participating in this trial had obtained Institutional Review Board approval, and all patients provided written informed consent prior to their participation.

\section{Patient Selection}

Only patients treated with cetuximab on N0147 through random assignment stratified by extent of lymph node involvement, tumor grade, and extent of tumor invasion were included. No other clinical or laboratory factors were utilized for assignment of therapy. Because this large phase III trial had been modified in June 2008 to allow randomization only of patients with K-raspositive tumors, it was decided that analyses for the present study would best be conducted by including only patients enrolled prior to this modification in an effort to circumvent any potential confounding effects of K-ras status on rash development.

Otherwise, eligibility criteria were based on those outlined in the study protocol and are briefly summarized as follows: (1) recent, completely resected stage III colon cancer with an operation that entailed an adequate lymph node dissection; (2) age $\geq 18$ years at enrollment, and (3) an Eastern Cooperative Oncology Group performance status of 2 or better.

Patients were excluded in the event of the following: (1) pregnant/nursing; (2) recipient of prior or non-protocol-specified concurrent chemotherapy/radiation for the recently diagnosed colon cancer; (3) previous exposure to an epidermal growth factor receptor inhibitor; (4) other major malignant diagnosis rendered either previously or concurrently; (5) notable peripheral neuropathy or allergy to platinum compounds, or (6) other major medical illnesses that would or could make the planned study treatment too risky.

\section{Protocol Therapy and Monitoring}

All patients were to be treated with 4 drugs: oxaliplatin, 5fluorouracil, leucovorin, or FOLFOX6, for 6 months, as previously described [10] with the addition of cetuximab $400 \mathrm{mg} / \mathrm{m}^{2}$ intravenously over $2 \mathrm{~h}$ on week 1 followed by $250 \mathrm{mg} / \mathrm{m}^{2}$ intravenously weekly thereafter throughout the duration of chemotherapy.

Predictors of Cetuximab-Induced Rash
Patients were assessed for adverse events every 2 weeks. The Common Terminology Criteria, version 3, were utilized for this purpose [11]. The protocol specified that the rash/desquamation skin toxicity reporting criteria be used. Only grade 3 or worse adverse events, including rash, were to be reported. For purposes of this study, only rash that occurred in the first month of therapy is reported. This approach is appropriate because rash typically occurs within 7-10 days after the initiation of an epidermal growth factor receptor inhibitor [1].

Cetuximab dose reductions or discontinuation based on adverse events were outlined in the protocol. A grade 1 or 2 rash allowed for continuation of treatment and did not require reporting on the study case report forms. However, a grade-3 rash prompted holding cetuximab for a maximum of 2 weeks and restarting at a dose of $150 \mathrm{mg} / \mathrm{m}^{2}$ upon improvement. A grade 4 rash required discontinuation of cetuximab. The protocol mandated that both grade 3 and 4 rashes be reported.

\section{Analyses}

The main goals of this study were to report the percentage of patients who developed a grade 3 or worse rash and to identify clinical factors that predicted rash development. The former was reported as a percentage with $95 \%$ confidence intervals. A logistic regression model that included gender, age ( $\geq 70$ years vs. $<70$ years), race, and performance score was constructed to assess the predictive effect of these variables on rash development. These variables were selected based on previous reports that described a potential association between these variables and rash development [12-14]. Results are reported even in the absence of a statistically significant association with rash. Univariate and multivariate logistic regression models were tested. In the case of age, a statistically significant finding prompted further analyses that looked at age as a continuous variable.

Baseline demographics are presented descriptively. Results from the logistic regression models are presented as odds ratios with $95 \%$ confidence intervals. A p value $<0.05$ is considered statistically significant.

\section{Results}

A total of 933 patients are the focus of this study. Demographics for the group as a whole are shown in table 1. The median age was 59 years (range: $22-86$ years). Slightly more patients were men (51\%), and most (86\%) were white.

Fifty patients (5\%) developed a grade 3 rash from cetuximab. No grade 4 rashes were reported. Of incidental note, among patients who did not receive cetuximab, no rashes were reported.

Severe rash among cetuximab-treated patients was more commonly observed in men and younger patients, and both univariate and multivariate analyses yielded identical conclusions (table 2). More men compared to women developed a grade 3 rash: $34(7 \%)$ versus $16(3 \%)$ (multivariate odds ratio $=2.10 ; 95 \%$ confidence inter- 
val: $1.14-3.88 ; \mathrm{p}=0.017)$. Similarly, a greater number of younger patients $(<70$ years of age) developed a grade 3 rash: $48(6 \%)$ versus $2(1 \%)$ (multivariate odds ratio $=0.21$; 95\% confidence interval: $0.05-0.88 ; \mathrm{p}=0.032$ ).

This age-related predictive effect led to an assessment of age as a continuous variable. Again, univariate and multivariate analyses provided consistent conclusions, and an inverse relationship between age and severe rash was observed (odds ratio: 0.96 for each year of age $(95 \%$ confidence interval: $0.93-0.98 ; \mathrm{p}=0.0005$ by multivariate analysis).

The risk of severe rash was additive. Between the two risk factors of sex and age, in men $<70$ years of age, the risk of severe rash was $8 \%$. In contrast to the above findings, race and performance score were not predictive of severe rash development.

After development of a severe rash, 27 of these 50 patients were able to restart cetuximab at a lower dose. Of these, 14 completed all 12 of the planned treatment cycles with this agent.

\section{Discussion}

To our knowledge, few previous studies have focused on identifying clinical predictors of severe rash from epidermal growth factor receptor inhibitors. This 933-patient study was undertaken for this purpose and observed that men and younger patients are at greater risk for severe rash. However, in both these high-risk groups, the absolute risk of severe rash is low $(<10 \%)$. Nonetheless, these findings suggest these patients should be counseled about the low but somewhat increased possibility of developing a severe rash from cetuximab. More importantly, knowledge of these risk factors is critical for the design of future rash palliative or prevention trials. Our results suggest that gender and age should be utilized as stratification factors in such trials.

Are these results plausible? Preclinical data suggest they are. With advancing age, cultured fibroblasts express fewer epidermal growth factor receptors, an observation that suggests that epidermal growth factor receptor inhibitors may have fewer cutaneous targets in older patients and hence less cutaneous toxicity in older patients [15].

Additionally, androgens and estrogens also appear to interact with the epidermal growth factor receptor [16], and these hormonal interactions imply that different rates of rash development based on gender carry a scientific basis. Hence, such preclinical observations add plausibility to the findings reported in the current study.

Table 1. Baseline demographics

$\begin{array}{lc}\text { Age } & \\ \text { Median } & 59 \\ \text { Range } & 22-86 \\ \geq 70 \text { years of age } & 149(16) \\ \quad<70 \text { years of age } & 784(84) \\ \text { Gender } & \\ \quad \text { Male } & 474(51) \\ \text { Female } & 459(49) \\ \text { Race } & \\ \text { White } & 803(86) \\ \text { African American } & 65(7) \\ \text { Asian } & 42(5) \\ \text { Other or unreported } & 23(2) \\ \text { Performance score } & \\ 0 & 694(74) \\ 1-2 & 239(26)\end{array}$

Numbers refer to numbers of patients listed, unless otherwise specified. Numbers in parentheses refer to percentages.

Table 2. Statistical analysis of the results

\begin{tabular}{|c|c|c|c|c|c|c|}
\hline \multirow[t]{2}{*}{ Factor } & \multicolumn{3}{|c|}{ Univariate analyses } & \multicolumn{3}{|c|}{ Multivariate analyses ${ }^{1}$} \\
\hline & $\begin{array}{l}\text { odds } \\
\text { ratio }\end{array}$ & $95 \% \mathrm{CI}$ & $\begin{array}{l}\mathrm{p} \\
\text { value }\end{array}$ & $\begin{array}{l}\text { odds } \\
\text { ratio }\end{array}$ & $95 \% \mathrm{CI}$ & $\begin{array}{l}\mathrm{p} \\
\text { value }\end{array}$ \\
\hline Male & 2.14 & $1.16-3.93$ & 0.014 & 2.10 & $1.14-3.88$ & 0.017 \\
\hline$\geq 70$ years old & 0.21 & $0.05-0.87$ & 0.001 & 0.21 & $0.05-0.88$ & 0.032 \\
\hline Non-white race ${ }^{2}$ & 1.44 & $0.55-3.76$ & 0.46 & 1.56 & $0.56-4.16$ & 0.37 \\
\hline Performance score 1 or $2^{3}$ & 0.91 & $0.47-1.78$ & 0.79 & 0.92 & $0.47-1.79$ & 0.80 \\
\hline \multicolumn{7}{|c|}{$\begin{array}{l}\mathrm{CI}=\text { Confidence interval. } \\
{ }^{1} \text { Analyses are adjusted for gender, age, and race. }{ }^{2} \text { White is the referent group. } \\
{ }^{3} \text { Performance score of } 0 \text { is the referent group. }\end{array}$} \\
\hline
\end{tabular}


It should be noted, however, that our results differ from those previously reported. First, in a 731-patient study with erlotinib in patients with metastatic nonsmall-cell lung cancer, a recently published secondary analysis reported that patients 70 years of age or older $(\mathrm{n}=112)$ developed a severe rash at a higher rate: $16 \%$ versus $6 \%(\mathrm{p}=0.003)$ in contrast to our findings in which a severe, cetuximab-induced rash occurred more often in younger patients [12]. Although it is difficult to reconcile the findings of this study with our results, it should be noted again that others have described an age-associated decrease in expression of the epidermal growth factor receptor and that these findings appear to be consistent with the findings reported in our study [15]. Second, Lai et al. [13] preliminarily reported on 42 patients and observed that lighter skin pigmentation was associated with higher rates of rash from erlotinib. Although our study did not evaluate skin pigmentation per se, we did not find that race predicted a severe cetuximab-induced rash. With regard to this latter point, it should be noted that despite this lack of association, we cannot totally discount the issue of skin color in these analyses, as skin color can vary considerably even among people of a specific race. Third, in contrast to Wacker et al. [14], who observed a lose association between performance score and rash development in 444 cancer patients, we did not observe any such association. To our knowledge, there is not yet a strong scientific basis to suggest that a better performance score is associated with rash. Thus, although a few of the observations reported here are divergent from those previously reported, such variability of results underscores the importance of further studying clinical predictors of rash induced by epidermal growth factor receptor inhibitors with the long-term goal of enabling us to better prevent or palliate this cutaneous side effect.

\section{Acknowledgements}

This study was conducted as a trial of the North Central Cancer Treatment Group and Mayo Clinic and was supported in part by Public Health Service grants CA-25225, CA-37404, and CA35195 from the National Cancer Institute Department of Health and Human Services. It was also supported by R21CA134432 and K24CA131099 to Aminah Jatoi, MD.

\section{References}

-1 Solomon BM, Jatoi A: Rash from EGFR inhibitors: opportunities and challenges for palliation. Curr Oncol Rep 2008;100:304308

$>2$ Scott J, Dakhil T, Cosgriff T, et al: Cetuximab + FOLFOX6 as first-line therapy for metastatic colorectal cancer. 2005 ASCO Annu Meet Proc. J Clin Oncol 2005;23(16S): 3705A.

3 Wagner LL, Lacouture ME: Dermatologic toxicities associated with EGFR inhibitors: the clinical psychologist's perspective. Impact on health-related quality of life and implications for clinical management of psychological sequelae. Oncology 2007;21(suppl 5):34-36.

4 Jatoi A, Nguyen PL: Do patients die from rashes from epidermal growth factor receptor inhibitors? A systematic review to help counsel patients about holding therapy. Oncologist 2008;13:1201-1204.

75 Scope A, Agero AL, Dusza SW, et al: Randomized double-blind trial of prophylactic oral minocycline and topical tazarotene for cetuximab-associated acne-like eruption. J Clin Oncol 2007;25:5390-5396.
6 Jatoi A, Rowland K, Sloan JA, et al: Tetracycline to prevent epidermal growth factor receptor inhibitor-induced skin rashes: results of a placebo-controlled trial from the North Central Cancer Treatment Group (N03CB). Cancer 2008;113:847-853.

7 Mitchell E, LaCouture M, Shearer H, et al: Updated results of STEPP, a phase 2, openlabel study of pre-emptive versus reactive skin toxicity treatment in metastatic colorectal cancer (mCRC) patients receiving panitumumab + FOLFIRI or irinotecan-only chemotherapy as second-line treatment. 10th World Congr Gastrointestinal Cancer, Barcelona, 2008.

8 Rudin CM, Liu W, Desai A, et al: Pharmacogenomic and pharmacokinetic determinants of erlotinib toxicity. J Clin Oncol 2008; 26:1119-1127.

9 Alberts SR, Sinicrope F, Grothey A: N0147: a randomized phase III trial of oxaliplatin plus 5 -fluorouracil/leucovorin with or without cetuximab after curative resection of stage III colon cancer. Clin Colorectal Cancer 2005;5:211-213.

10 Maindrault-Goebel F, Louvet C, André T, et al: Oxaliplatin added to the simplified bimonthly leucovorin and 5-fluorouracil regimen as second-line therapy for metastatic colorectal cancer (FOLFOX6). Eur J Cancer 1999;35:1338-1342.
11 http://ctep.cancer.gov/forms/CTCAEv3.pdf; last accessed November 5, 2008.

12 Wheatley-Price P, Ding K, Seymour L, et al: Erlotinib for advanced non-small cell lung cancer in the elderly: an analysis of the $\mathrm{Na}$ tional Cancer Institute of Canada Clinical Trials Group Study BR.21. J Clin Oncol 2008; 26:2350-2357.

13 Lai SE, Minnelly L, O’Keeffe P, et al: Influence of skin color in the development of erlotinib-induced rash: a report from the SERIES clinic. 2007 ASCO Annu Meet Proc, part I. J Clin Oncol 2007;25(suppl 18S): 9127a.

14 Wacker B, Nagrani T, Weinberg J, et al: Correlation between development of rash and efficacy in patients treated with the epidermal growth factor receptor tyrosine kinase inhibitor in two large phase III studies. Clin Cancer Res 2007;13:3913-3921.

15 Reenstra WR, Yaar M, Gilchrest BA: Aging effects epidermal growth factor receptor phosphorylation and traffic kinetics. Exp Cell Res 1996;227:252-255.

16 Migliaccio A, Castoria G, Di Domenico M, et al: Crosstalk between EGFR and extranuclear steroid receptors. Ann NY Acad Sci 2006; 1089:194-200. 